\title{
Selective Requirement of Pax6, But Not Emx2, in the Specification and Development of Several Nuclei of the Amygdaloid Complex
}

\author{
Shubha Tole, ${ }^{1}$ Ryan Remedios, ${ }^{1}$ Bhaskar Saha, ${ }^{1}$ and Anastassia Stoykova ${ }^{2}$ \\ ${ }^{1}$ Department of Biological Sciences, Tata Institute of Fundamental Research, Mumbai 400005, India, and 2Max-Planck Institute of Biophysical Chemistry, \\ D-37077 Göttingen, Germany
}

The amygdaloid complex is a group of nuclei that are thought to originate from multiple sites of the dorsal and ventral telencephalic neuroepithelium. The mechanisms that regulate their development are essentially unknown. We studied the role of Pax6 and Emx2, two transcription factors that regulate regional specification and growth of the telencephalon, in the morphogenesis of the amygdaloid complex. We used a set of specific marker genes that identify distinct amygdaloid nuclei to analyze Pax6/Small eye and Emx2 knock-out mutant mouse brains. We found that there is a selective requirement for Pax6, but not Emx2, in the formation a subset of nuclei within the amygdaloid complex. Specifically, structures that were not previously considered to be developmentally linked, the nucleus of the lateral olfactory tract and the lateral, basolateral, and basomedial nuclei, all appear to have a common requirement for Pax6. Together, our findings provide new insights into the origins and mechanisms underlying the development of the amygdaloid complex.

Key words: amygdala; telencephalon; PSB; specification; patterning; nLOT

\section{Introduction}

The mechanisms that parcellate the telencephalon are a subject of great current interest. From early stages, the telencephalic neuroepithelium is divided into domains that express distinct combinations of transcription factors. This parcellation has provided a mechanism for the specification of different telencephalic structures and cell types (Wilson and Rubenstein, 2000). The patterning of the telencephalon is beginning to be understood in terms of distinct pallial and subpallial domains. Going from dorsal to ventral, the medial (MP), dorsal (DP), lateral (LP), and ventral pallium (VP) (Puelles et al., 2000) constitute the dorsal telencephalic domains. The ventral telencephalon contains the medial ganglionic eminence (MGE) and the lateral ganglionic eminence (LGE) as well as a region defined as the dorsal LGE (dLGE), which is located at the extreme edge of the LGE (Yun et al., 2001). The LP, VP, and dLGE are of particular interest because they are located at the pallium-subpallium boundary (PSB) and are proposed to generate "interface" structures that eventually reside quite distant from the original domains in the neuroepithelium. These structures [the claustrum, endopiriform nucleus, amygdaloid com-

Received July 23, 2004; revised Jan. 20, 2005; accepted Jan. 20, 2005.

This work was supported by a Wellcome Trust Senior Fellowship to S.T. (056684/Z/99/Z) and by the Max-PlankGesellschaft to A.S. We thank I. Bach (Clim1a), R. Kageyama (NeuroD), G. Lemke (SCIP), Y. Nakagawa (Lhx9), F. D. Porter (Lhx2), and T. Rabbitts (Lm03) for gifts of plasmid DNA. The technical assistance of S. Eckert and M. Daniel is highly acknowledged.

Correspondence should be addressed to either of the following: Shubha Tole, Department of Biological Sciences, Tata Institute of Fundamental Research, Mumbai 400005, India, E-mail: stole@tifr.res.in; or Anastassia Stoykova, Max-Planck Institute of Biophysical Chemistry, Am Fassberg, D-37077 Göttingen, Germany, E-mail: astoyko@gwdg.de.

DOI:10.1523/JNEUROSCI.3014-04.2005

Copyright $\odot 2005$ Society for Neuroscience $\quad 0270-6474 / 05 / 252753-08 \$ 15.00 / 0$ plex, and olfactory cortex (Bayer and Altman, 1991; Puelles et al., 2000; Tamamaki et al., 2001; Gorski et al., 2002)] are the least understood in terms of the mechanisms that control their specification and development.

Pax6 plays a significant regulatory role in dorsoventral patterning (Stoykova et al., 1996, 2000), and mutations of this gene affect the specification of the ventral pallium, which takes on a subpallial identity (Stoykova et al., 2000; Yun et al., 2001). Furthermore, cell migration is disrupted in the absence of Pax6 function (Chapouton et al., 1999). Previous studies have reported defects in the formation of the piriform cortex, claustrum, endopiriform nucleus, and anterior amygdala in Pax6 mutant brains (Stoykova et al., 2000). These findings raised the hypothesis that the amygdaloid complex, thought to arise in part from the LP and VP, may be disrupted in the Pax6 mutant because of either a specification defect or a migration defect.

Pax6 is thought to exert a complementary role to that of Emx2 in the dorsal telencephalon. This idea is based on the observation of opposing expression gradients of these two genes (Bishop et al., 2000) and that each is required for the graded expression of the other: Pax6 and Emx2 inhibit the expression of each other, such that in the Pax6 mutant, the Emx2 gradient is flattened out and vice versa (Muzio et al., 2002; Muzio and Mallamachi, 2003). Furthermore, Pax6 and Emx2 are suggested to act as heterodimers where they are coexpressed (Bishop et al., 2002; Muzio et al., 2002).

Studies of the roles of Pax6 and Emx2 to date have focused primarily on the preneurogenic neuroepithelium from which the cortex arises; however, there are reports of Pax6 expression marking what appears to be a stream of cells arising from the palliumsubpallium boundary (Toresson et al., 2000; Yun et al., 2001). 
This region is thought to contribute to the amygdaloid complex (Smith-Fernandez et al., 1998; Puelles et al., 1999, 2000; Molnar and Butler, 2002). Here, we confirm and extend previous reports of multiple transcription factors identifying streams of cells in the lateral telencephalon. Furthermore, we establish the expression of these genes in the final locations of different amygdaloid nuclei. Finally, we examine the mechanisms involved in the development of the amygdaloid complex.

A complete analysis of the development of the amygdala has been hampered by a lack of markers for distinct amygdaloid nuclei. We used a comprehensive panel of markers (Remedios et al., 2004) to assess the roles of Pax6 and Emx2 in the development of this structure. We found a selective requirement for Pax6, but not $E m \times 2$, in the formation of specific amygdaloid nuclei, revealing distinct functions for these genes in the development of this complex structure.

\section{Materials and Methods}

Timed pregnant Swiss mice were obtained from the Tata Institute animal breeding facility. Pax6 and Emx2 mutant embryos were obtained by intercrossing mice heterozygous for the Small eye mutant, Sey allele (Roberts, 1967; Hogan et al., 1986) and heterozygous Emx2+/- mutants, respectively (Pellegrini et al., 1996), which were genotyped before use. All procedures followed Institute Animal Ethics Committee guidelines and National Institutes of Health guidelines for the care and use of animals. In situ hybridization was performed as described previously (Bulchand et al., 2003). Briefly, the hybridization was performed overnight at $70^{\circ} \mathrm{C}$ in $5 \times$ SSC, $50 \%$ formamide, and $1 \%$ SDS. Posthybridization washes were at $70^{\circ} \mathrm{C}$ in $2 \times$ SSC and $50 \%$ formamide and $1 \%$ SDS. Foster's atlas (1998) was used as a guide to the morphology of the embryonic amygdaloid complex and for nomenclature as follows: anterior amygdaloid area (AA); AA, dorsal portion (AAD); AA, ventral portion (AAV); the various "nuclei": anterior cortical (ACo); basolateral (BL); basomedial (BM); central (Ce); I (intercalated nucleus); lateral (La); medial (Me); Me, posterodorsal portion $(\mathrm{MeD})$; Me, posteroventral portion $(\mathrm{MeV})$; nucleus of the lateral olfactory tract (nLOT).

Images were grabbed using an Axiocam attached to an Axioskop2 plus, via Axiovision software. False color overlays were made as in Yun et al. (2001). Briefly, images of serial sections were "inverted" in Adobe Photoshop (version 7.0; Adobe Systems, San Jose, CA) to give a white-onblack picture, which was imported through either the red or green channels. These pseudocolored pictures were then overlaid.

\section{Results}

Molecularly distinct streams of cells arise at the PSB and head toward the region where the amygdaloid complex will form

As a starting point for our studies, we examined the expression patterns of transcription factors in the lateral aspect of the embryonic day 12.5 (E12.5) telencephalon. This is the stage at which Pax6, Tbr1, Emx1, and Lhx2 have been reported to identify streams of cells that appear to arise at the PSB and extend toward prospective amygdalar regions (Puelles et al., 2000; Yun et al., 2001; Medina et al., 2004). We examined the expression of some of these markers together with that of $L h \times 9$ and $E m \times 2$.

The first interesting feature of the gene expression patterns is that there is an upregulation or downregulation of specific genes between the ventricular zone (VZ) and the adjacent region in the mantle where streams of postmitotic cells are seen. Thus, three transcription factors, $P a x 6, E m \times 2$, and $L h \times 2$, are expressed in the ventricular zone in a continuous stretch in the region of the PSB. The Pax6 domain includes the dLGE, whereas $L h \times 2$ and Emx2 remain restricted to the pallial domains (Fig. $1 a, c, g, i$ ). In the adjacent mantle, these and two other transcription factors, Lhx9 and Tbr1, identify streams of cells in a combinatorial manner as follows. Pax6 expression is confined to a thin band of cells that constitutes the dLGE (Puelles et al., 2000; Yun et al., 2001). This stream extends toward the basal telencephalon, terminating at the olfactory tuberculum (Puelles et al., 2000) (Fig. 1a,g).

In adjacent sections from the same brain, the expression of the LIM-HD transcription factor Lhx2 marks only the VP migrating stream (Fig. 1c) (Yun et al., 2001). Similarly, the expression domain of another LIM-HD molecule, $L h x 9$ overlaps very well with that of $L h \times 2$ (Fig. 1b,c). Both $L h \times 2$ - and $L h \times 9$-expressing cells fall within a domain of weak Tbr1 expression (Fig. $1 d, f$ ). Tbrl expression is reported to be weak in the VP and in the adjacent LP and strong in the more lateral DP (Puelles et al., 2000). When the patterns of Pax 6 or Tbr 1 are overlaid with that of $L h x 9$, it is seen that the $L h \times 2 / 9$-positive stream appears perfectly complementary to the Pax6-positive domain (Fig. 1e). That the Pax6-positive domain is juxtaposed to but excludes the Tbr1/Lhx9-expressing domain is further confirmed by two-color in situ hybridization, in which Pax6 (blue) is distinct from either $\operatorname{Lh} \times 9$ or Tbrl staining (brown)(Fig. 1l,m)

Emx2 also identifies a stream of cells emerging from the PSB. In adjacent sections from the same brain, it appears that $E m \times 2$ labeling overlaps well with $L h x 9$ in the more peripheral portion of the migrating stream, whereas expression of $E m \times 2$ nearer the ventricular zone is sparse (Fig. $1 g-k$ ). Emx2 expression, like that of $\operatorname{Lh} x 9$, also appears perfectly complementary to that of Pax6 (Fig. 1j).

In summary, these results reveal a molecular parcellation within the migratory streams in the basolateral telencephalon, confirming and extending the previously reported schema (Puelles et al. 2000; Yun et al. 2001; Medina et al., 2004), with new information presented here about the expression profiles of $\operatorname{Lh} x 9$ and $E m \times 2$. Within the migrating streams, distinct domains can be distinguished going from lateral to medial (Fig. 1, schematic); the most lateral is the DP, which intensely expresses Tbr1. Next is the LP, which shows weak Tbr1 expression; then the VP, which is positive for Tbr1 (weak), $L h \times 9, \operatorname{Lh} \times 2, E m \times 2$; and finally, a most medial domain, the dLGE, a subpallial domain expressing only Pax6.

\section{Identification of distinct amygdaloid nuclei at late embryonic stages corresponding to the early migrating streams}

To study which amygdalar structures might arise from the dLGE and VP migrating streams observed at E12.5, we followed the expression patterns of $\operatorname{Pax} 6, E m \times 2, L h \times 2$, and $L h \times 9$ at later stages, when amygdaloid nuclei are distinguishable in their final locations, using Tbr 1 as a reference marker. Several interesting observations emerged. Pax6 and the VP stream markers Emx2, Lhx2, and $L h x 9$ continue to identify mutually exclusive structures. Rostrally, Pax6 intensely labels the AAD and the Ce (Fig. 2b). Emx2, $L h x 2$, and $L h x 9$ are coexpressed in the adjacent AAV and the ACo (Fig. $2 c-e$ ). Further caudally, Pax6 identifies the intercalated nucleus (Fig. 2h), which does not express Emx2, Lhx2, or $L h \times 9$. This is consistent with the interpretation that this nucleus may be partially subpallial in origin (Medina et al., 2004). Thus, similar to stage E12.5, the expression of Pax6 and Emx2, $L h \times 2$, and $L h \times 9$ in late embryonic amygdalar nuclei also display complementarity of expression (Fig. 2f,l). This supports the idea that the respective nuclei may arise from the corresponding migratory streams.

The nLOT is identified by intense expression of Tbr1 (Fig. 2a-e) (Medina et al., 2004). Pax6 and Emx2 do not label this nucleus, whereas $L h \times 2$ and $L h x 9$ are expressed only in layer 1 of the nLOT (Fig. 2a-e) (Remedios et al., 2004). Tbr1 also marks the La, BL, and BM at more caudal levels (Puelles et al., 2000) (Fig. $2 g$ ). At this level of sectioning, the expression patterns of Emx2, 


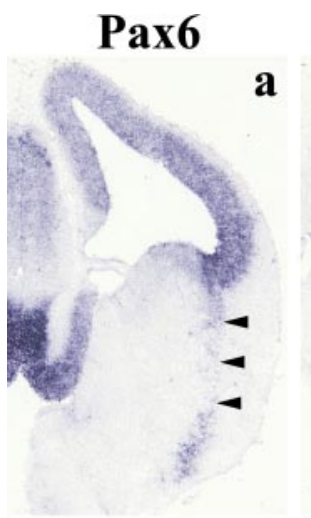

Lhx9

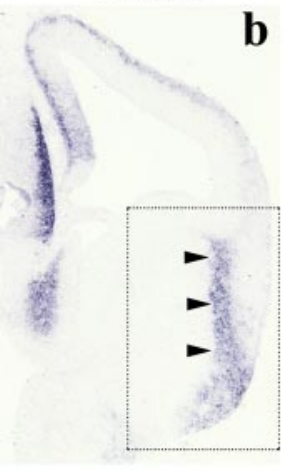

Lhx2

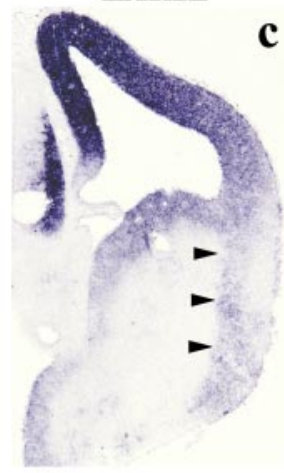

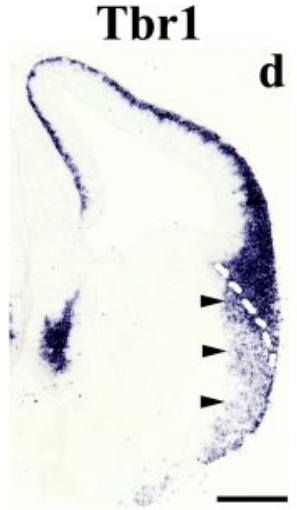

Lhx9Pax6

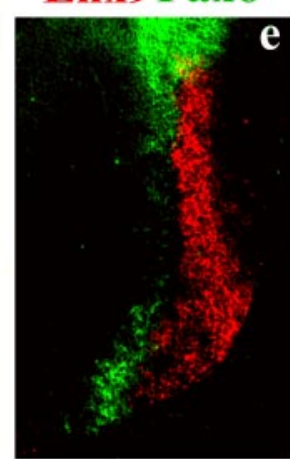

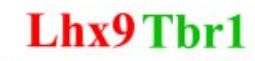

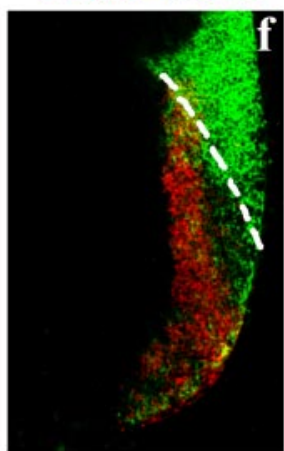

Pax6
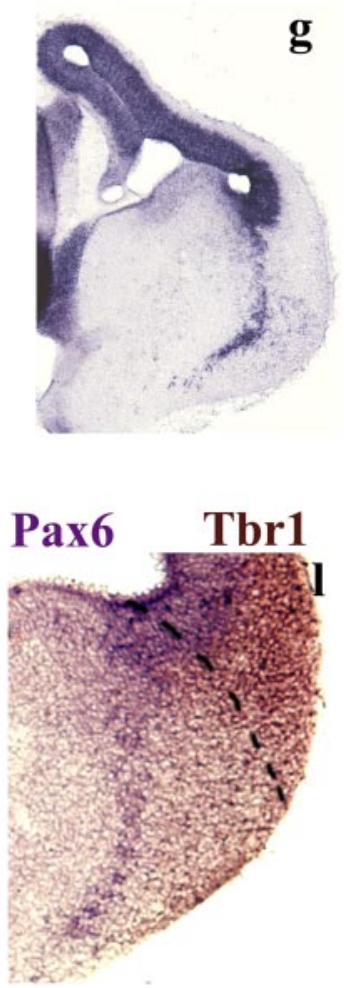

Lhx9

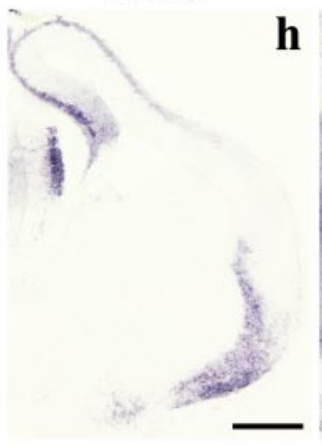

Emx2

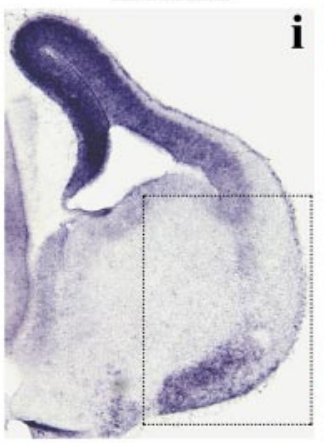

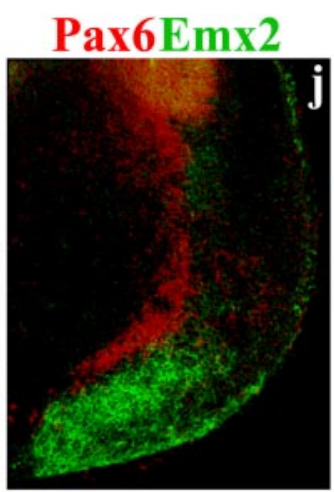

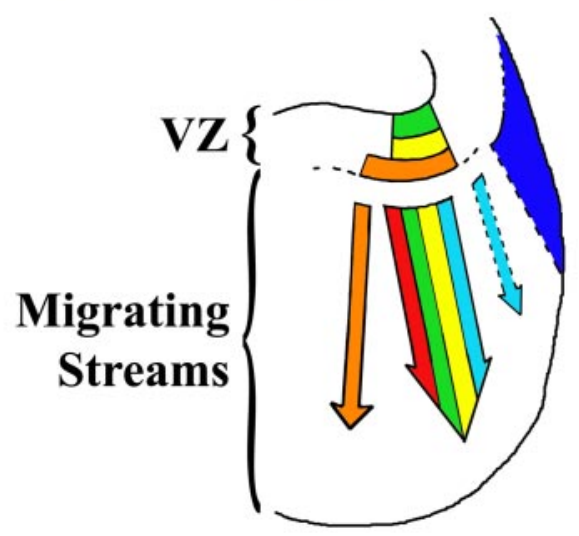

Lhx9 Emx2
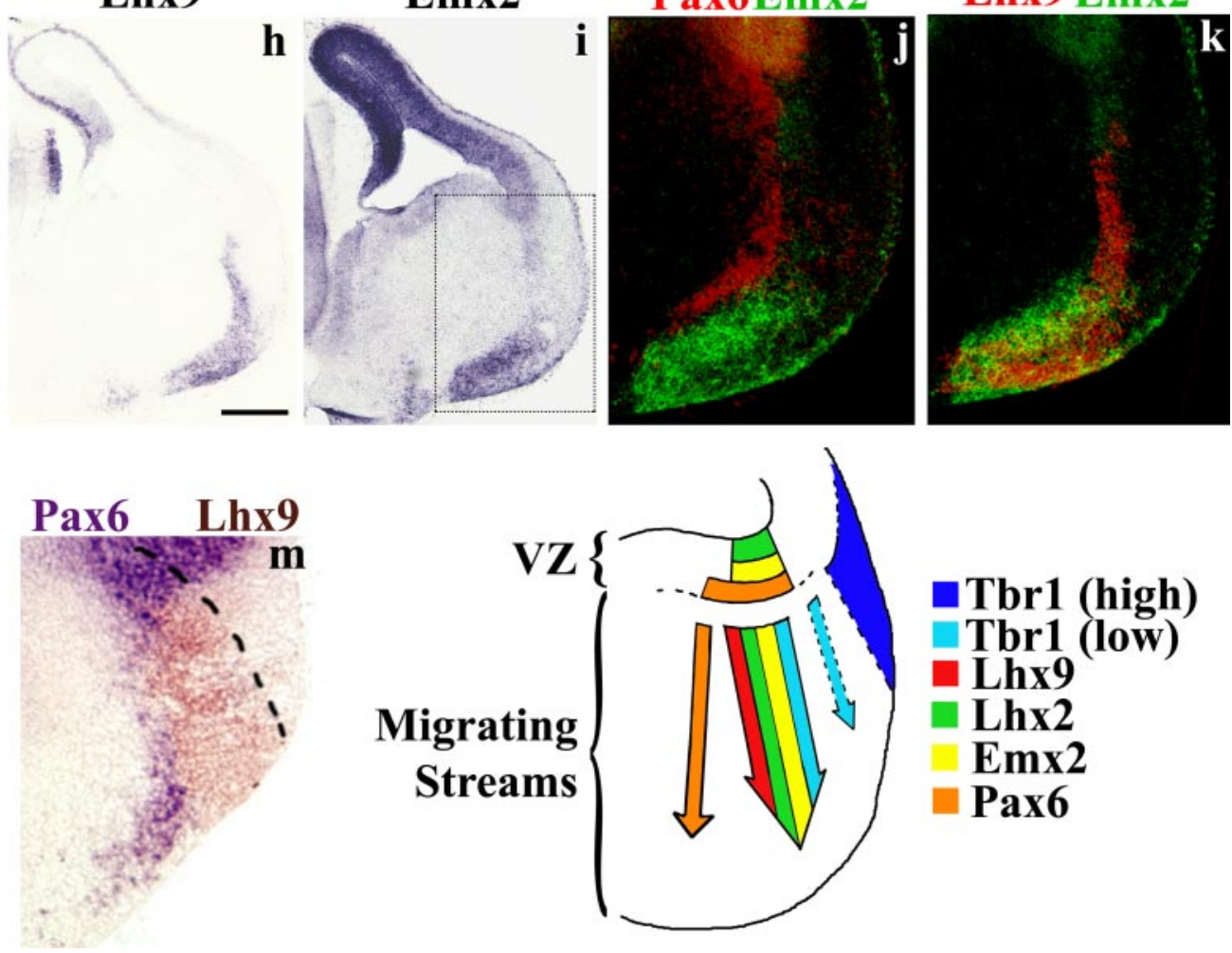

Figure 1. Distinct streams of cells emerge from the PSB and extend toward the amygdaloid complex. At E12.5, serial sections from the same brain display expression of Pax6 (a), Lhx 9 (b), Lhx2 $(\boldsymbol{c})$, and $\operatorname{Tbr} 1(\boldsymbol{d})$ in migrating streams in the basolateral telencephalon ( $\boldsymbol{a}-\boldsymbol{d}$, arrowheads). The expression of Pax6 is complementary to $L h \times 9$, as seen in a false color overlay of the two patterns ( $\boldsymbol{e}$, which is an overlay of $\boldsymbol{a}$ and $\boldsymbol{b}$ ). A false color overlay $(\boldsymbol{f})$ illustrates the overlap of $L h \times g(\boldsymbol{b})$ with the weakly expressing portion of the Tbr1 expression domain, delineated by dashed lines $(\boldsymbol{d}, \boldsymbol{f})$. Lhx 9 expression appears similar to that of $L h \times 2$ (compare $\boldsymbol{b}, \boldsymbol{c})$. Serial sections from a different brain reveal complementary expression of Pax6 and Emx2, using $L h \times 9$ expression as a reference $(\boldsymbol{g}-\boldsymbol{i})$. This complementarity is confirmed using false color overlays ( $\boldsymbol{j}$, which is an overlay of $\boldsymbol{g}$ and $\boldsymbol{h}$ ). Lhx9 expression, in contrast, overlaps well with Emx2 ( $\boldsymbol{k}$, which is an overlay of $\boldsymbol{h}$ and $\boldsymbol{i})$. Two-color in situ hybridization demonstrates that $\operatorname{Pax} 6$ expression $(\boldsymbol{I}, \boldsymbol{m}$, blue) abuts but does not overlap with that of either $\operatorname{Tbr} 1(\boldsymbol{I}$, brown) or $L h \times 9$ ( $\boldsymbol{m}$, brown). Scale bar, $250 \mu \mathrm{m}$. The expression of different markers in the VZ and in the migrating streams is summarized in the schematic. The DP and LP (dark blue and light blue) are indicated for completeness, although this study focuses on the VP and dLGE.

Lhx2, and Lhx9 begin to diverge. Emx2 is expressed only in the Me; $L h x 9$ is expressed in the $\mathrm{BM}$ and $\mathrm{MeV}$; $\operatorname{Lh} x 2$ is weakly expressed in scattered cells in the BL and BM (Fig. 2i-k).

In summary, on the one hand, some direct correlations can be observed between the expression of molecular markers of the different migrating streams at E12.5 with that seen in late embryonic amygdaloid nuclei. On the other hand, some of the expression patterns indicate that there is no simple correspondence between the molecular parcellation within the ventricular zone, the migrating streams at early stages, and the discrete structures seen in the ventrolateral telencephalon at later embryonic stages. Together, the data indicate an intricate upregulation and down- regulation of transcription factors in the different stages of development of amygdaloid structures: in the ventricular zone, in the migratory stream, and in the final location of the nucleus. This motivates a careful analysis of the functions of these molecules in the embryonic amygdala.

The amygdalar phenotype in the Sey/Sey mutant

To explore the role of Pax6 and Emx2 in the development of the amygdaloid complex, we examined Pax6-/- (Sey/Sey) and Emx2-/- brains at two stages: first, at E12.5, the stage at which molecularly distinct migrating streams are seen in the lateral telencephalon, and at later stages (E15.5-E17.5), when distinct 

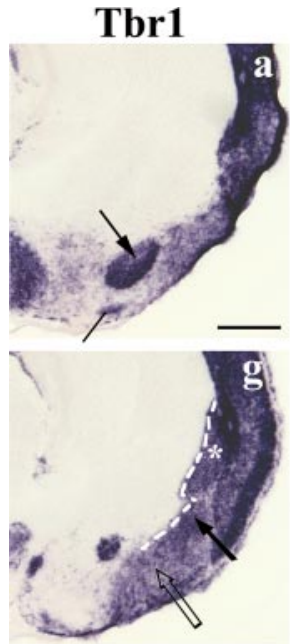

Pax6
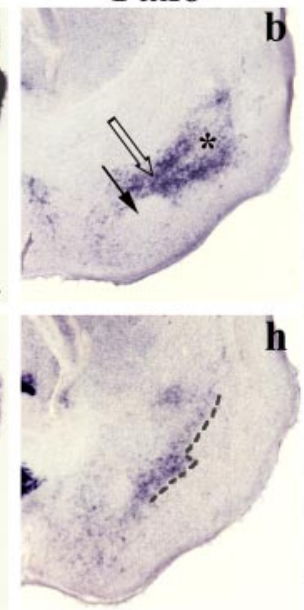

Emx2
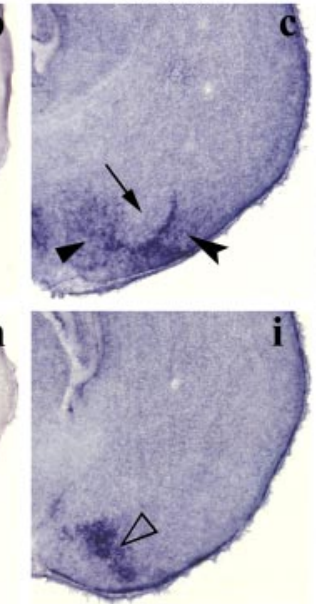

Lhx9

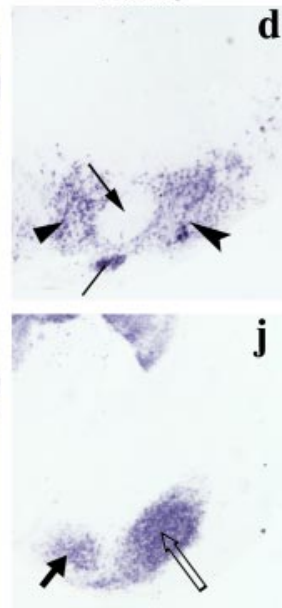

d
Lhx2
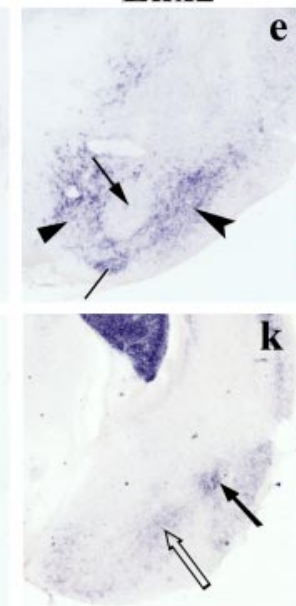

e

k
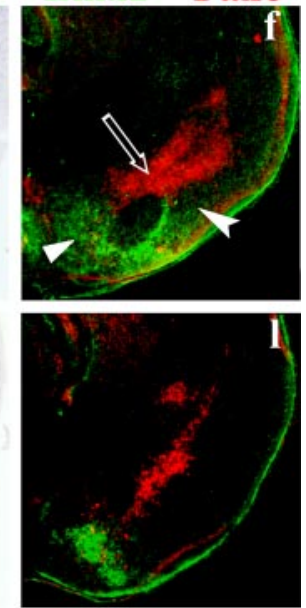

Figure 2. Expression of molecular markers in distinct amygdaloid nuclei at late embryonic stages. At E16.5 $(\boldsymbol{a}-\boldsymbol{c}, \boldsymbol{g}-\boldsymbol{i})$ and E17.5 (d, $\boldsymbol{e}, \boldsymbol{j}, \boldsymbol{k})$, serial sections from the same brain reveal mutually exclusive expression of Pax $6(\boldsymbol{b}, \boldsymbol{h})$ and $T b r 1, E m \times 2, L h \times 2$, and $L h \times 9(\boldsymbol{a}, \boldsymbol{c}-\boldsymbol{e}, \boldsymbol{g}, \boldsymbol{i}-\boldsymbol{k})$ in the amygdaloid complex from mid to caudal levels. Pax6 expression is seen in the AAD and the $C$ ( $(\boldsymbol{b}, 0$ pen arrow and asterisk, respectively). Tbr1, Emx2, Lhx2, and $L h \times 9$ label the AAV and the ACo ( $\boldsymbol{a}, \boldsymbol{c}-\boldsymbol{e}$, arrowhead and notched arrowhead, respectively). 0 nly $T b r 1$ is expressed in the $n L 0 T 2 / 3$ ( $\boldsymbol{a}-\boldsymbol{e}$, thin arrow).

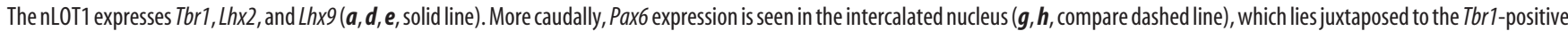
La ( $\boldsymbol{g}$, white asterisk), BL ( $\boldsymbol{g}$, thick arrow), and BM ( $g$, open arrow). Emx2 expression marks the Me (i, open arrowhead), which is excluded by both Tbr 1 and Pax6. Lhx 9 expression is seen in the BM $(\boldsymbol{j}$, open arrow) and in the MeV $(\boldsymbol{j}$, short arrow). Weak and scattered $L h x 2$ expression is seen in the BL ( $\boldsymbol{k}$, long arrow) and in the BM ( $\boldsymbol{k}$, open arrow). The mutually exclusive expression of Pax6 and Emx2 is illustrated in false color overlays ( $\boldsymbol{f}$, an overlay of $\boldsymbol{b}$ and $\boldsymbol{c} ; \boldsymbol{I}$, an overlay of $\boldsymbol{h}$ and $\boldsymbol{i}$ ). Scale bar, $250 \mu \mathrm{m}$.

amygdaloid nuclei can be identified. At E12.5, we examined the expression of Pax6 transcript. Consistent with previous reports, Pax6 transcript expression is reduced in the neuroepithelium of the VP and dLGE (Stoykova et al., 2000). Furthermore, the migrating stream emerging from the dLGE is not apparent in the mutant, suggesting that the cells fail to migrate in the absence of Pax6 (Fig. 3a,e). Strikingly, this defect appears limited to the dLGE migrating stream. Lhx9, Emx2, or Tbr1 expression identifies the VP and LP migrating streams in the Sey/Sey mutant (Fig. $3 b-d, f-h$ ). This indicates that the presence of functional Pax6 in the neuroepithelium of the VP and LP is not critical to generating streams of migrating cells from these domains.

At older stages, Sey/Sey mutants reveal a disruption of several amygdaloid structures. Previous morphological analysis indicated a severe dysgenesis of the amygdaloid complex in E18.5 Sey/Sey brain, where some structures such as the claustrum and endopiriform nucleus could not be distinguished (Stoykova et al., 2000). Here, we examine discrete anterior amygdaloid structures and basolateral structures of the Sey/Sey mutant using specific markers at E17.5.

Starting with anterior structures, three independent markers, NeuroD, a bHLH transcription factor, SCIP, a POU-domain transcription factor, and LIM gene Lmo3, all mark the nLOT 2/3 in control brains (Fig. 4a,b,d) (Remedios et al., 2004). These layers of the nLOT appear to be missing in the Sey/Sey mutant (Fig. 4a,b,d). Interestingly, the superficial layer of the nLOT (nLOT1), which expresses $L h x 9$, appears to be normally specified and positioned in the Pax6 mutant (Fig. 4c), suggesting that this layer of the nLOT may be regulated by different, Pax6-independent mechanisms.
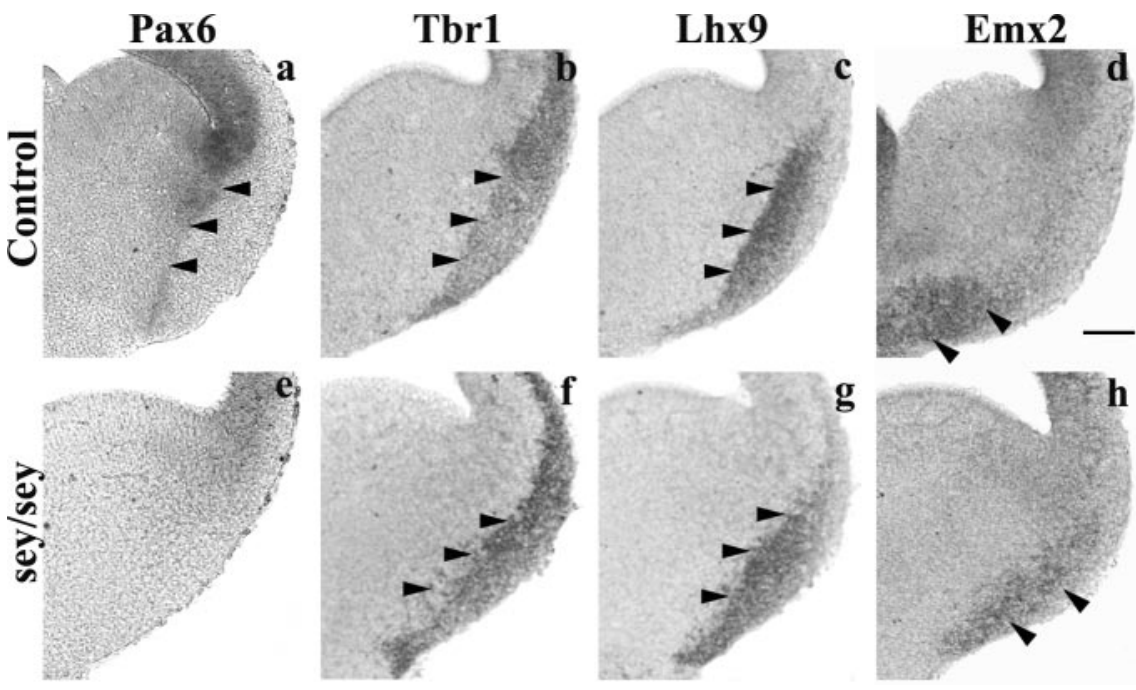

Figure 3. Selective perturbation of the migrating streams in the absence of Pax6. Sections of control and Pax6 mutant E12.5 brains reveal a decrease in Pax6 expression in the VZ of the mutant PSB, and the dLGE stream of cells is missing $(\boldsymbol{a}, \boldsymbol{e})$. In contrast, expression of $T b r 1$ is seen in the LP and VP migrating streams ( $\boldsymbol{b}, \boldsymbol{f}$, arrowheads), and $L h \times 9$ (c, $\boldsymbol{g}$, arrowheads) and Emx2 (d, $\boldsymbol{h}$, arrowheads) expression is seen in the VP stream of both control and mutant sections. Scale bar, $100 \mu \mathrm{m}$.

Lhx9 also labels the AAV and ACo and reveals dysmorphology of these structures in the Pax6 mutant. The labeled regions appear shrunken and also displaced toward the pial surface in the mutant compared with the control (Fig. 4c).

During examination of more caudal levels, Clim1a and Lmo3, which label the La and BL in control brains, are not detectable in the corresponding regions of the Pax 6 mutant brains, although expression in other structures such as the neocortex and striatum (Clim1a) and globus pallidus (Lmo3) appears normal (Fig. 5a,c). The BM, ventral to the basolateral complex, appears drastically shrunken, as revealed by Clim $1 a$ and Lhx9 expression (Fig. 5a,d). To test whether these structures were properly specified at earlier stages but possibly became defective over time, we also examined E15.5 brains, the earliest stage at which specific marker expres- 

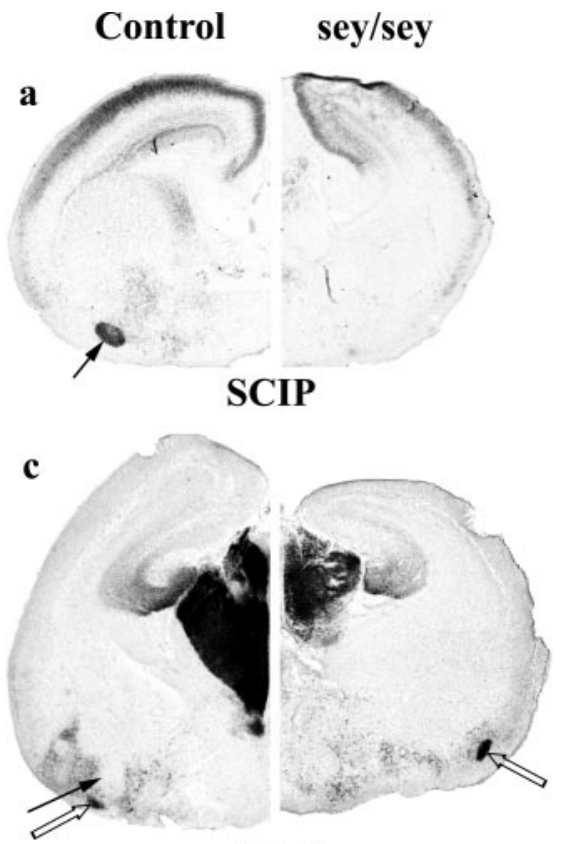

Lhx9

Figure 4. Pax6 is necessary for the specification of the $\mathrm{nLOT} 2 / 3$. Sections at E17.5 reveal expression of SCIP, NeuroD, and $L m 03$ in layers $2 / 3$ of the $n L 0 T$ of control but not Pax6 mutant brains $(\boldsymbol{a}, \boldsymbol{b}, \boldsymbol{d}$, arrow). Other structures, such as the globus pallidus (d, asterisk), are detected in the mutant by $L m 03$ expression. $L h x 9$ expression excludes the $n L 0 T 2 / 3(c$, arrow) but marks the nLOT1, which is preserved in the Pax6 mutant (c, open arrow). Lhx 9 is also expressed in the AAV and the $A C$, which lie on either side of the $\mathrm{nL} 0 \mathrm{~T}$. The Pax6 mutant displays expression in scattered cells expressing $L h x 9$ at a corresponding location (c). Scale bar, $500 \mu \mathrm{m}$.

sion is seen (Remedios et al., 2004). The basolateral complex is missing at this age as well, and the basomedial nucleus also appears extremely reduced (Fig. $5 e, f$ ).

In contrast to the anterior and lateral structures, medial structures within the amygdaloid complex appear relatively less affected in the Pax6 mutant. Climla expression in the MeD and $\mathrm{MeV}$ is seen in the appropriate locations in control and mutant brains (Fig. $5 b$ ). This indicates that the specification and morphological formation of these nuclei do not require Pax6. The small reduction in size of these nuclei is consistent with the slightly smaller size of the Pax6 mutant brain compared with control.

Using the same set of markers, we examined the effect of loss of function of transcription factor $\mathrm{Emx} 2$ in developing Emx2-1- embryos. Surprisingly, no detectable perturbation of the amygdaloid complex was seen. At E17.5, the mutant nLOT expresses $L m o 3$ in layers 2/3, whereas $L h x 9$ expression is seen in layer 1 , as in the control (Fig. $6 a, c$ ). $L h \times 9$ is also expressed in the mutant and control ACo and AAV (Fig. $6 c$ ). The mutant La and BL both strongly express $\operatorname{Lmo3}$ (Fig. 6b); the BM expresses $L h x 9$ strongly, whereas the $\mathrm{MeV}$ shows weaker $\operatorname{Lh} x 9$ expression, as in controls (Fig. 6d).

\section{Discussion}

It is generally accepted that the amygdalar structures originate from the pallial and subpallial neuroepithelium flanking the PSB, located at the junction of the dorsal and ventral telencephalon. Several authors have reported streams of cells that arise from the PSB and progress in a ventral direction. At different points in the pathway, accumulations of cells named the "head" and the "reservoir" have been identified by Bayer et al. (1991), from which cells migrate and contribute to formaiton of the piriform cortex and other ventrolateral structures. Recent evidence indicates that the $\mathrm{La}, \mathrm{BL}$, and $\mathrm{BM}$ amygdaloid nuclei are pallial in origin, arising from the VP and LP, respectively (Puelles et al., 2000; Medina et al., 2004); however, the migrating streams from each of these domains have yet to be definitively fate mapped.

Several transcription factors are implicated in cell-fate specification in the telencephalon, and mutations in these cause selective regional defects or perturb specific processes such as migration (Wilson and Rubenstein, 2000). Analysis of the effects of these mutations on amygdaloid development has not been possible because of a dearth of markers that identify the various amygdaloid nuclei. Furthermore, several patterning-defective mutants die in embryonic stages, making the analysis of their possible roles in the development of this structure more difficult. We used a newly established set of markers that selectively label amygdaloid nuclei in embryonic stages (Remedios et al., 2004) to evaluate the roles of two important players in dorsoventral telencephalic patterning, Pax6 and Emx2, in the development of this structure.

The genes Pax6 and Emx2, encoding homeodomain transcription factors, have been implicated as selector genes for cortical fate and suppressors of subpallial fate (Muzio et al., 2002). In the developing cortex, Pax6 and Emx2 are expressed in opposite gradients in the telencephalic neuroepithelium. Loss of function of either of these genes causes severe and complementary defects in cortical area specification, consistent with their expression gradients (Bishop et al., 2000). We hypothesized that Pax6 and Emx2 also regulate the development of the amygdala, because it is a complex structure consisting of numerous nuclei extending in a large rostrocaudal domain within the basolateral telencephalon.

The most significant findings in this study are that in the Pax6 mutant, the dLGE migrating stream is absent, and a large number of amygdaloid nuclei are missing or extremely reduced. In contrast, in the Emx2 mutant, the amygdaloid complex appears normal. Several interesting and surprising aspects emerge from these findings. First, the lack of Pax6 expression at embryonic stages in nuclei that are disrupted in the mutant suggests a role for Pax6 in the VZ or during migration of the cells. Second, the structures disrupted in the Pax6 mutant include nuclei from different subgroups: the nLOT, together with the ACo, are thought to be olfactory structures and considered to be part of the olfactory cortex, a pallial derivative, although their precise neuroepithelial origin is not known (Swanson and Petrovich, 1998). The La, BL, and $\mathrm{BM}$ are thought to be part of a different pallial amygdaloid subgroup, arising from the VP and LP (Puelles et al., 2000; Medina et al., 2004). Not much is known about the origin or affiliation of AA, but it is thought to have a mixed pallial and subpallial origin, whereas the medial and central nuclei form the "subpallial” group (Swanson and Petrovich, 1998; Medina et al., 2004). Our findings suggest a common requirement for Pax6 across subgroups, raising the possibility of multiple roles for Pax6 after subgroup identity has been allocated. Finally, in the cortex, Pax6 and Emx2 mutant phenotypes exhibit a complementarity that is 

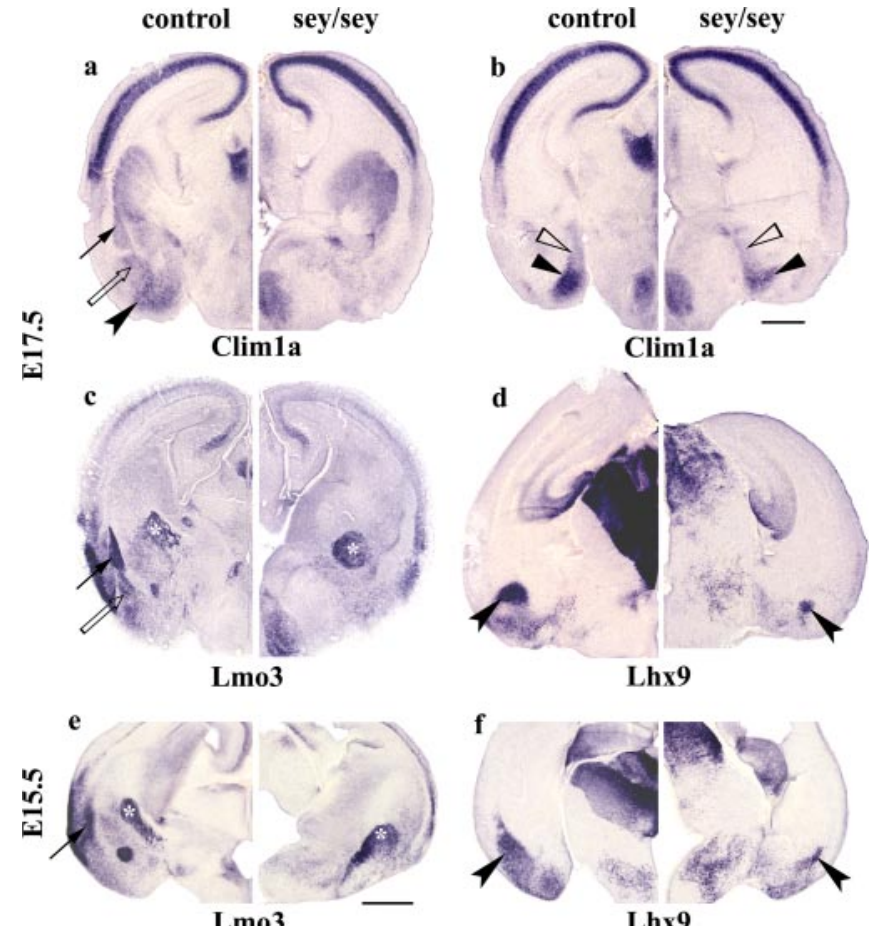

Lhx9

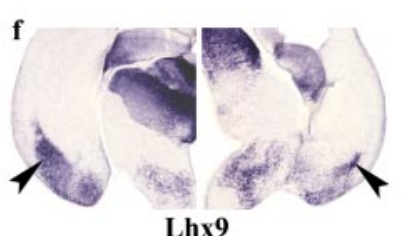

Figure 5. The lateral amygdaloid structures are severely disrupted in the absence of Pax6, but medial and caudal structures appear less affected. Sections at E17.5 at mid levels probed for expression of Clim 1a (a) and Lmo3 (c) display expression in the La (arrow) and the BL (open arrow) of control but not Pax6 mutant brains. Clim $1 a$ and Lhx9 expression is seen in the BM of control brains but identifies only a shrunken structure in the appropriate location in the Pax6 mutant $(\boldsymbol{a}, \boldsymbol{d}$, notched arrowhead). Sections at E15.5 reveal similar results, with $L$ mo3 expression identifying the control but not the mutant $L a(\boldsymbol{e}$, arrow) and $L h \times 9$ expression revealing a greatly reduced BM in the mutant $(\boldsymbol{f}$, notched arrowhead). In contrast, both portions of the medial nucleus, MeD and MeV (open arrowhead and arrowhead, respectively), express Clim $1 a$ in control and mutant brains $(\boldsymbol{b})$. Asterisk in $\boldsymbol{c}$ and $\boldsymbol{e}$ indicates the globus pallidus, marked by Lmo3 expression. Scale bar, $500 \mu \mathrm{m}$.
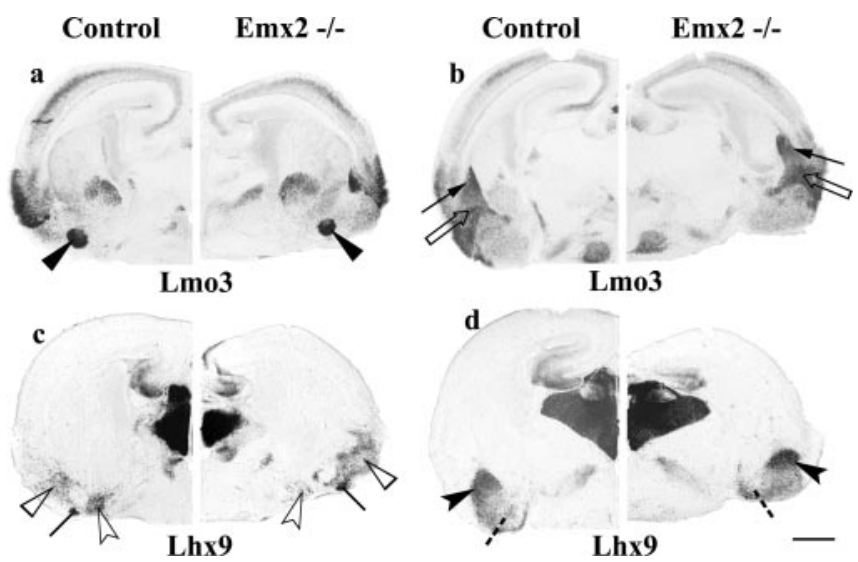

Figure 6. Emx2 is not required for the development of the amygdaloid complex. All examined markers reveal similar expression in control and Emx2 mutant brains. At mid levels, $L m 03$ expression identifies the $\mathrm{nL} 0 \mathrm{~T} 2 / 3$ ( $\boldsymbol{a}$, arrowhead), and $L h \times 9$ expression is seen in the $\mathrm{nLOT1}$ ( $\boldsymbol{c}$, solid line), the AAV ( $\boldsymbol{c}$, notched open arrowhead), and the ACo ( $\boldsymbol{c}$, open arrowhead). At more caudal levels, Lmo3 labels the La and BL ( $\boldsymbol{b}$, arrow and open arrow, respectively). Lhx 9 expression is seen in the BM ( $\boldsymbol{d}$, notched arrowhead) and the MeV (d, dashed line). Scale bar, $500 \mu \mathrm{m}$.

consistent with their expression gradients (Bishop et al., 2000). In the amygdala, however, we found no such complementary phenotypes, suggesting that the cooperative actions of Pax6 and Emx2 in this complex may be quite different from those in the cortex. These issues will be discussed in detail below.
Although Pax6 expression in the wild-type E12.5 telencephalon is present in the ventricular zone of dLGE, VP, and LP and also in the dLGE migratory stream, it is not detectable at later stages in the nuclei that are affected in the mutant, the nLOT, La, $\mathrm{BL}$, or BM. Therefore, Pax6 must function at earlier stages in these cells, either during the specification of these cells in the ventricular zone or during their migration. That the lateral migratory stream is defective in the absence of Pax6 has been noted previously, and cells were seen to continue migrating to accumulate at the pial surface (Brunjes et al., 1998). Suggesting a basis for defective migration in the absence of Pax6, these mutants also reveal altered expression of the cell adhesion molecules L1, neural cell adhesion molecule, and R-cadherin (Caric et al., 1997; Stoykova et al., 1997; Simpson and Price, 2002). The defects in the Pax6 mutant amygdaloid nuclei could potentially be explained as a failure of the cells to migrate to their correct positions and assemble into morphologically recognizable structures. This scenario of a migration defect would imply that these cells are specified correctly but would be mislocated to the pial surface if they have over-migrated or may be found near their origins at the ventricular zone if they have under-migrated; however, using several independent markers, we detected no such mislocated cells. These observations indicate that the primary defect in the Pax6 mutant amygdala is that of specification or proliferation of the precursors that will contribute to specific amygdaloid nuclei.

There is as yet no good understanding of where and when the cells destined for distinct amygdaloid nuclei are specified, in contrast to the cortex, in which layer-specific identity is known to be established in the ventricular zone (McConnell and Kaznowski, 1991); however, there are some indications of the origins of different nuclei. One suggestion is that at very rostral levels, the Pax6-positive dLGE stream may contribute to the formation of the olfactory tubercle, the nucleus accumbens, and the primordium of the anterior olfactory nucleus (Puelles et al., 2000). Furthermore, the rostral VP neuroepithelium has been proposed to be the site of origin of the ventromedial claustrum, La, endopiriform nucleus, and ACo (Puelles et al., 2000; Medina et al., 2004). Some of these structures appear to be missing in the Pax6 mutant brain (Stoykova et al., 2000). It is striking that Pax6 expression levels are highest in the rostrolateral neuroepithelium (Bishop et al., 2000; Stoykova et al., 2000), a region that includes the rostral $\mathrm{VP}$ and dLGE domains. Our findings of severe disruptions in the nLOT2/3, La, and BL of Sey/Sey mutants suggest that high Pax6expressing regions give rise to structures that are strongly affected in the absence of Pax6. In contrast, we find the BM to be less severely affected (shrunken, but not missing) in the absence of Pax6. This nucleus is thought to arise from the caudal VP (Medina et al., 2004), a region of low Pax6 expression (Bishop et al., 2000). Similarly, the Me is thought to arise from the VZ of the subpallium (Stenman et al., 2003), a region of extremely low Pax6 expression (Stoykova et al., 2000), and may be expected to have a weak phenotype. Indeed, we find normal morphology and marker expression in the $\mathrm{MeD}$ and $\mathrm{MeV}$. In summary, similar to the lateral cortex (Stoykova et al., 2000; Yun et al., 2001) and the spinal cord (Burrill et al., 1997; Ericson et al., 1997), the severity of the Pax6 mutant phenotype within the amygdaloid complex appears to depend on the level of Pax6 expression in the neuroepithelial domain from which the different structures arise.

Striking specification defects in the neuroepithelium of the Pax6 mutant were reported previously; a general feature of the Pax6 phenotype was a dorsal shift of subpallial markers (Stoykova et al., 2000; Toresson et al., 2000, Yun et al., 2001). In particular, the expression of genes normally restricted to the 
MGE, Nxk2.1, and shh spreads into the LGE (Stoykova et al., 2000). One possibility is that the perturbations in the amygdaloid complex are a result of the altered levels of shh experienced by the cells, because the shh expression domain has spread laterally. A more fundamental cause, however, seems to be a specification defect of the neuroepithelium. Several studies indicate that the dLGE, together with the VP and LP, shows the strongest phenotype in the absence of functional Pax6: Mash1, Dlx1,2, and Gsh2, which are normally restricted to the MGE and LGE neuroepithelium, spread dorsally into VP-LP, whereas VP-LP markers Ngn2, Tbr1, and $L h \times 2$ are almost eliminated at the PSB (Stoykova et al., 1996, 2000; Toresson et al., 2000; Yun et al., 2001; Muzio et al., 2002). Rather than being shifted dorsally, the VP in the Pax6 mutant appears to be defective, based on the absence of VPspecific markers sFrp2 and Dbx1 (Kim et al., 2001; Yun et al., 2001; Assimacopoulos et al., 2003). A deficient VP in the Tlx mutant has been correlated with disrupted La and BL development (Stenman et al., 2003).

Our report that the Pax6 mutant dLGE fails to generate a migrating stream of cells at E12.5 places this structure in an important position as a possible source to many amygdaloid nuclei, together with the VP and LP. Interestingly, although the dLGE is a subpallial structure, it appears not to contribute to the Me and AAV, both of which are present in the Pax6 mutant. This raises the question of what structures the normal dLGE stream contributes to and suggests a possible role for the dLGE in the formation of the "pallial" nuclei nLOT2/3, La, BL, and BM. Pax6 may function in the VZ of the dLGE, VP, and LP to regulate specification and/or proliferation of the cells that will populate the nLOT2/3, $\mathrm{La}, \mathrm{BL}$, and BM. Finally, this is the first report of distinct regulatory mechanisms underlying the formation of different layers of an amygdaloid nucleus, the nLOT, such that layers $2 / 3$ require Pax6 function but layer 1 is Pax6 independent.

In striking contrast to the disrupted amygdaloid complex in the Pax6 mutant is the apparently normal amygdaloid complex in the Emx2 mutant. In the cortex, the loss of Emx2 causes a dramatic shift in area boundaries (Bishop et al., 2002) and a disruption of hippocampal morphogenesis (Tole et al., 2000), whereas there does not appear to be any effect of the loss of this protein on amygdaloid development. This may be because of redundancy with Emx1, as has been suggested and tested in the cortex (Bishop et al., 2003; Muzio and Mallamachi, 2003). Analysis of the Emx1/2 double knock-out may reveal a role for these genes in the amygdala. Supporting this idea is the report of Gorski et al.(2002), in which cells of the Emx1 lineage populate the La and BL.

An alternative hypothesis for the apparently normal Emx2 mutant amygdala is based on the levels of expression of Emx2 in the PSB region. Although Pax6 is expressed in a high rostrolateral, low caudomedial gradient, Emx2 has a complementary expression (Bishop et al., 2000). Each of these mutations may display the strongest phenotype in regions where the gene is most highly expressed. Consistent with this idea, it is the medial-most structure of the hippocampus, the dentate gyrus, that displays a strong morphological disruption in the absence of Emx2 (Pellegrini et al., 1996; Yoshida et al., 1997; Tole et al., 2000). The PSB is a region of low Emx2 expression, which may explain why its derivatives are spared in the mutant. In contrast, for Pax6, the phenotypes may be expected to correlate with its level of expression in the rostral versus the caudal PSB domains, as has been discussed above.

In summary, our findings reveal no complementarity in the amygdalar phenotype between the Pax6 and Emx2 mutants, indicating that any interplay between Pax6 and Emx2 in the speci- fication of the amygdaloid complex is likely to be different from their interactions in regulating regional domains within the VZ. Additional studies will examine whether the amygdaloid phenotype in the Pax6 mutant is enhanced in the absence of one or both copies of $E m \times 2$. This will test for a cooperative role between the two molecules, as has been shown in the cortical primordium (Bishop et al., 2000). In addition, Pax6 is known to cooperate with other molecules such as Gsh2 and Tlx in specifying the pallium-subpallium boundary (Toresson et al., 2000; Stenman et al., 2003), and these interactions may also play a role in the development of the amygdaloid complex. This study used a panel of markers that will be a useful tool for analyzing these questions and could reveal molecular mechanisms of specification that may be unique to the development of the amygdala.

\section{References}

Assimacopoulos S, Grove EA, Ragsdale CW (2003) Identification of a Pax6dependent epidermal growth factor family signaling source at the lateral edge of the embryonic cerebral cortex. J Neurosci 23:6399-6403.

Bayer SA, Altman J (1991) Development of the endopiriform nucleus and the claustrum in the rat brain. Neuroscience 45:391-412.

Bayer SA, Altman J, Russo RJ, Dai XF, Simmons JA (1991) Cell migration in the rat embryonic neocortex. J Comp Neurol 307:499-516.

Bishop KM, Goudreau G, O’Leary DD (2000) Regulation of area identity in the mammalian neocortex by Emx2 and Pax6. Science 288:344-349.

Bishop KM, Rubenstein JL, O'Leary DD (2002) Distinct actions of Emx1, Emx2, and Pax6 in regulating the specification of areas in the developing neocortex. J Neurosci 22:7627-7638.

Bishop KM, Garel S, Nakagawa Y, Rubenstein JL, O’Leary DD (2003) Emx1 and Emx2 cooperate to regulate cortical size, lamination, neuronal differentiation, development of cortical efferents, and thalamocortical pathfinding. J Comp Neurol 457:345-360.

Brunjes PC, Fisher M, Grainger R (1998) The small-eye mutation results in abnormalities in the lateral cortical migratory stream. Brain Res Dev Brain Res 110:121-125.

Bulchand S, Subramanian L, Tole S (2003) Dynamic spatiotemporal expression of LIM genes and cofactors in the embryonic and postnatal cerebral cortex. Dev Dyn 226:460-469.

Burrill JD, Moran L, Goulding MD, Saueressig H (1997) PAX2 is expressed in multiple spinal cord interneurons, including a population of EN1+ interneurons that require PAX6 for their development. Development 124:4493-4503.

Caric D, Gooday D, Hill RE, McConnell SK, Price DJ (1997) Determination of the migratory capacity of embryonic cortical cells lacking the transcription factor Pax-6. Development 124:5087-5096.

Chapouton P, Gartner A, Gotz M (1999) The role of Pax6 in restricting cell migration between developing cortex and basal ganglia. Development 126:5569-5579.

Ericson J, Rashbass P, Schedl A, Brenner-Morton S, Kawakami A, van Heyningen V, Jessell TM, Briscoe J (1997) Pax6 controls progenitor cell identity and neuronal fate in response to graded Shh signaling. Cell 90:169-180.

Foster GA (1998) Chemical neuroanatomy of the prenatal rat brain. Oxford: Oxford UP.

Gorski JA, Talley T, Qiu M, Puelles L, Rubenstein JL, Jones KR (2002) Cortical excitatory neurons and glia, but not GABAergic neurons, are produced in the Emx1-expressing lineage. J Neurosci 22:6309-6314.

Hogan BL, Horsburgh G, Cohen J, Hetherington CM, Fisher G, Lyon MF (1986) Small eyes (Sey): a homozygous lethal mutation on chromosome 2 which affects the differentiation of both lens and nasal placodes in the mouse. J Embryol Exp Morphol 97:95-110.

Kim AS, Anderson SA, Rubenstein JL, Lowenstein DH, Pleasure SJ (2001) Pax-6 regulates expression of SFRP-2 and Wnt-7b in the developing CNS. J Neurosci 20:RC132(1-5).

McConnell SK, Kaznowski C (1991) Cell cycle dependence of laminar determination in developing neocortex. Science 254:282-285.

Medina L, Legaz I, Gonzalez G, De Castro F, Rubenstein JL, Puelles L (2004) Expression of Dbx1, Neurogenin 2, Semaphorin 5A, Cadherin 8, and Emxl distinguish ventral and lateral pallial histogenetic divisions in the developing mouse claustroamygdaloid complex. J Comp Neurol 474:504-523. 
Molnar Z, Butler A (2002) The corticostriatal junction: a crucial region for forebrain development and evolution. BioEssays 24:530-541.

Muzio L, Mallamachi A (2003) Emx1, Emx2 and Pax6 in specification, regionalization and arealization of the cerebral cortex. Cereb Cortex 13:641-647.

Muzio L, DiBenedetto B, Stoykova A, Boncinelli E, Gruss P, Mallamaci A (2002) Emx2 and Pax6 control regionalization of the pre-neuronogenic cortical primordium. Cereb Cortex 12:129-139.

Pellegrini M, Mansouri A, Simeone A, Boncinelli E, Gruss P (1996) Dentate gyrus formation requires Emx2. Development 122:3893-3898.

Puelles L, Kuwana E, Puelles E, Rubenstein JL (1999) Comparison of the mammalian and avian telencephalon from the perspective of gene expression data. Eur J Morphol 37:139-150.

Puelles L, Kuwana E, Puelles E, Bulfone A, Shimamura K, Keleher J, Smiga S, Rubenstein JL (2000) Pallial and subpallial derivatives in the embryonic chick and mouse telencephalon, traced by the expression of the genes Dlx-2, Emx-1, Nkx-2.1, Pax-6, and Tbr-1. J Comp Neurol 424:409-438.

Remedios R, Subramanian L, Tole S (2004) LIM genes parcellate the embryonic amygdala and regulate its development. J Neurosci 24:6986-6990.

Roberts RC (1967) Small-eyes: a new dominant mutant in the mouse. Genet Res 9:121-122.

Simpson TI, Price DJ (2002) Pax6: a pleiotropic player in development. BioEssays 24:1041-1051.

Smith-Fernandez AS, Pieau C, Reperant J, Boncinelli E, Wassef M (1998) Expression of the Emx-1 and Dlx-1 homeobox genes define three molecularly distinct domains in the telencephalon of mouse, chick, turtle and frog embryos: implications for the evolution of telencephalic subdivisions in amniotes. Development 125:2099-2111.

Stenman J, Yu R, Evans R, Campbell K (2003) Tlx and Pax6 co-operate genetically to establish the pallio-subpallial boundary in the embryonic mouse telencephalon. Development 130:1113-1122.

Stoykova A, Fritsch R, Walther C, Gruss P (1996) Forebrain patterning defects in small eye mutant mice. Development 122:3453-3465.

Stoykova A, Gotz M, Gruss P, Price J (1997) Pax6-dependent regulation of adhesive patterning, R-cadherin expression and boundary formation in developing forebrain. Development 124:3765-3777.

Stoykova A, Treichel D, Hallonet M, Gruss P (2000) Pax6 modulates the dorsoventral patterning of the mammalian telencephalon. J Neurosci 20:8042-8050.

Swanson LW, Petrovich GD (1998) What is the amygdala? Trends Neurosci 21:323-331.

Tamamaki N, Nakamura K, Kaneko T (2001) Cell migration from the corticostriatal angle to the basal telencephalon in rat embryos. NeuroReport 12:775-780.

Tole S, Goudreau G, Assimacopoulos S, Grove EA (2000) Emx2 is required for growth of the hippocampus but not for hippocampal field specification. J Neurosci 20:2618-2625.

Toresson H, Potter S, Campbell K (2000) Genetic control of dorsal-ventral identity in the telencephalon: opposing roles for Pax6 and Gsh2. Development 127:4361-4371.

Wilson SW, Rubenstein JL (2000) Induction and dorsoventral patterning of the telencephalon. Neuron 28:641-651.

Yoshida M, Suda Y, Matsuo I, Miyamoto N, Takeda N, Kuratani S, Aizawa S (1997) Emx1 and Emx2 functions in development of dorsal telencephalon. Development 124:101-111.

Yun K, Potter S, Rubenstein JL (2001) Gsh2 and Pax6 play complementary roles in dorsoventral patterning of the mammalian telencephalon. Development 128:193-205. 